\title{
Garcinia cambogia extract removes calcium oxalate kidney stones in both genetic and non-genetic Drosophila models of nephrolithiasis
}

Qiuxia Fan ${ }^{1 \#}$, Xiaoming Feng ${ }^{1 \#}$, Xizhen Hong ${ }^{1}$, Siqiao Gong ${ }^{1}$, Jianwei Tian ${ }^{1}$, Fanfan Hou ${ }^{1,2^{\star}}$, Fujian Zhang ${ }^{1,2^{*}}$

${ }^{1}$ Division of Nephrology, Nanfang Hospital, Southern Medical University, State Key Laboratory of Organ Failure Research, Guangzhou 510515, Guangdong, P. R. China

${ }^{2}$ Division of Nephrology, Nanfang Hospital, Southern Medical University, State Key Laboratory of Organ Failure Research, National Clinical Research Center of Kidney Diseases, Guangzhou 510515, Guangdong, P. R. China

\# These authors contributed equally to this work

* To whom correspondence should be addressed:

Fujian Zhang, PhD

Nanfang Hospital, Southern Medical University

1838 North Guangzhou Blvd.

Guangzhou 510515, Guangdong, P. R. China

Phone: +86-020-62786323

Email: flykidney@163.com

or Fanfan Hou, PhD MD

Nanfang Hospital, Southern Medical University

1838 North Guangzhou Blvd.

Guangzhou 510515, Guangdong, P. R. China

Phone: +86-020- 61641591

Email: ffhouguangzhou@163.com 


\begin{abstract}
Kidney stone formers with family history have a high rate of stone recurrence after kidney stone removal surgery and there is no effective medication available for treatment. Here, we show that Garcinia cambogia extract (GCE) efficiently removes calcium oxalate kidney stones from Malpighian tubules in both genetic and non-genetic Drosophila models of nephrolithiasis, and hydroxycitrate -a major component of GCE, directly dissolves calcium oxalate stones in Drosophila Malpighian tubules ex vivo. Our study discovers a potential novel therapeutic strategy for the clinical treatment of nephrolithiasis and suggests that clinical-grade Garcinia cambogia extract could be used to treat patients with nephrolithiasis in the future.
\end{abstract}

Key words: Garcinia cambogia extract; Hydroxycitrate; Calcium oxalate kidney stone; Nephrolithiasis; Drosophila genetic model; Malpighian tubule 


\section{INTRODUCTION}

The incidence of nephrolithiasis, also known as kidney stone, is increasing globally and puts a huge burden on health care system gradually. Kidney stones often cause hematuria and severe pain in the groin, abdomen, or flank. Calcium-containing kidney stones are the most common type of kidney stone and account for more than $90 \%$ of renal stone diseases (Gee et al., 2016). Although almost 50\% of calcium oxalate (CaOx) stone formers are idiopathic(Ramaswamy et al., 2015, Chi and Stoller, 2011), kidney stones have long been thought to be the result of the interaction between genetic and environmental factors, including dehydration stemming from low fluid intake and high dietary intake of animal protein and salts. Meanwhile, more than 30 genes up to date have been identified as novel monogenic causes of kidney stone disease using whole exome sequencing (Gee et al., 2016, Halbritter et al., 2015, Braun et al., 2016, Daga et al., 2018, Sayer, 2017, Dowen et al., 2014). Effective medications are generally lacking, and the most advanced treatment for kidney stones is minimally invasive kidney stone surgical procedures, such as shockwave lithotripsy (SWL), ureteroscopy and laser lithotripsy and percutaneous nephrolithotomy (PCNL) depending on the size and type of stones. Despite great improvement in surgical techniques used to remove kidney stones in the last two decades, patients very often experience a high rate of stone recurrence after surgery, especially those who are inherited cases. The high rate of stone recurrence and the surgical burden associated with these patients demand the discovery of new drugs that could dissolve kidney stones in situ. However, no major progress of new drug discovery has been made to 
remove or prevent kidney stone formation in the last 30 years, mainly due to the lack of ideal animal models feasible for high-throughput drug screening.

The Drosophila excretory system is composed of nephrocytes and Malpighian tubules(Zhang and Chen, 2014). Our previous study showed that the Drosophila nephrocyte shares remarkable similarity with the glomerular podocyte for protein ultrafiltration, and the renal proximal tubule for protein reabsorption(Gee et al., 2015, Zhang and Chen, 2014, Zhang et al., 2013, Zhang et al., 2013). On the other hand, the Drosophila Malpighian tubule shares striking similar features with mammalian renal tubules and collecting ducts in terms of cell composition, anatomical structure and physiological function(Dow and Romero, 2010). There are two types of cells in Drosophila Malpighian tubule, the principal cells and stellate cells which contain many ion and organic solute transporters (Miller et al., 2013). The principal cells are the major tubular cell type $(\sim 80 \%)$ through which cations and organic solutes are transported. The stellate cells are the minor tubular cell type ( 20\%) through which chloride ion and water flow, and interspersed at regular intervals with the principal cells. They generate urine through active transport of ions, water and organic solutes from the hemolymph into the Malpighian tubule lumen(Dow and Romero, 2010). It has been shown that Drosophila Malpighian tubule is a powerful translational model system to study the pathogenesis of human nephrolithiasis, because one can easily generate the disease model, observe stones and perform genetic manipulation (Chen et al., 2017, Wu et al., 2014, Miller et al., 2013, Hirata et al., 2012a, Knauf and Preisig, 2011, Chi et al., 2015). Calcium oxalate stones are formed in Malpighian tubule with the addition of lithogenic 
agents in fly food within two weeks and can be directly examined under polarized light microscopy (Chen et al., 2011). Slc26a6 functions as a $\mathrm{Cl}^{-} / \mathrm{HCO}^{-}$exchanger and regulates oxalate secretion in human renal tube. Mutations of Slc26a6 have been identified from patients with nephrolithiasis. It also has been shown that RNAi knockdown of dPrestin specifically in principal cells, the Drosophila homolog of Slc26a6, led to decreased calcium oxalate stone formation in Malpighian tubules(Landry et al., 2016). These results strongly suggest that fruit fly could be an ideal genetic kidney stone disease model to screen novel genes involved in the pathogenesis of nephrolithiasis and validate the function of candidate genes identified from patients with nephrolithiasis in vivo(Hirata et al., 2012a).

The Drosophila calcium oxalate stone model has been used to screen traditional Chinese medicinal plants for the treatment of nephrolithiasis (Ho et al., 2013). Chen et al showed that commercial juices, such as apple, cranberry, orange, and pomegranate juices, failed to prevent calcium oxalate crystal formation in Drosophila nephrolithiasis model. Wu et al showed that some traditional Chinese Medicine (TCM) herbs have a potential antilithic effect, but it is still unclear whether they can directly dissolve kidney stones in situ to prevent kidney stone recurrence. In the last 30 years, no major progresses have been made in this field. Garcinia cambogia is a tropical fruit that grows in Southeast Asia and has been historically used for cooking in India. Garcinia cambogia extract containing $60 \%$ hydroxycitric acid (HCA), is a popular weight-loss supplement and sold at most health supplement and drug stores. Chung et al showed that HCA induces dissolution of the calcium oxalate crystal in vitro, suggesting that Garcinia cambogia has the potential as a novel treatment for calcium oxalate kidney 
stone(Chung et al., 2016). In this study, we used the Drosophila genetic nephrolithiasis model to test whether Garcinia cambogia and hydroxycitrate can prevent the formation of calcium oxalate stones in vivo. We found that Garcinia cambogia extract prevented calcium oxalate kidney stone formation and completely removed calcium oxalate kidney stones preformed in adult renal tubules in vivo. To further elucidate the molecular mechanism through which GCE removed calcium oxalate stones from renal tubules, we showed that hydroxycitrate - the major component of GCE - directly dissolved calcium oxalate stones in renal tubules ex vivo. Our data strongly suggest that clinical-grade Garcinia cambogia extract could be used to remove calcium oxalate renal stones in patients with nephrolithiasis.

\section{RESULTS}

\section{Garcinia cambogia extract efficiently prevents calcium oxalate kidney stone formation in Drosophila renal tubules in vivo.}

To test whether Garcinia cambogia can prevent the formation of calcium oxalate stones in vivo, $\mathrm{w}^{1118}$ wild type flies were reared in fly food containing $0.1 \% \mathrm{NaOx}$ and different concentrations of GCE for one week, and then the effect of GCE on the formation of kidney stone in renal tubules was examined. We found that Garcinia cambogia extract prevented calcium oxalate kidney stone formation in adult renal tubules in vivo in a concentration-dependent manner (Fig.1A-D). Compared with hydroxycitrate or citrate, Garcinia cambogia extract prevented the formation of calcium oxalate stone in Drosophila renal tubules at a very low concentration of $0.3 \%$ (Fig.1E). On the other hand, HCA or citric acid (CA) only partially blocked the formation of 
calcium oxalate stone in Drosophila renal tubules at a high concentration of $1.5 \%$ (Fig.1E), and both of them prevented calcium oxalate kidney stone formation in Drosophila renal tubules at a very high concentration of $3 \%$ (Fig.1E). These results indicate that Garcinia cambogia extract are a better reagent to prevent calcium oxalate stone formation in kidney stone disease models in vivo than citric acid which is widely used in clinic.

\section{Garcinia cambogia extract completely removes calcium oxalate kidney stones from adult Drosophila renal tubules in vivo.}

Previous study showed that hydroxycitric acid (HCA) can induce the dissolution of the calcium oxalate crystal in vitro. We reasoned that Garcinia cambogia extract (GCE) which contains $60 \%$ HCA could play a similar role in vivo. To test the effect of GCE on the removal of calcium oxalate renal stones in vivo, we first developed a calcium oxalate kidney stone disease model by feeding wild type flies with fly food containing $0.3 \% \mathrm{NaOx}$ for one week. Flies were then transferred to fly food containing $0.1 \% \mathrm{NaOx}$ and different concentrations of GCE for another week, and then the effect of GCE on the removal of kidney stone from renal tubules was examined. As shown in Figure 2, treatment with different concentrations of GCE for one week removed calcium oxalate kidney stones from adult renal tubules in a concentration-dependent manner (Fig.2AD). The total stone area decreased by about $93 \%$ in renal tubules of flies reared in fly food with $0.3 \%$ GCE compared to flies reared in $0.1 \% \mathrm{NaOx}$ only. No stones were left in renal tubules of flies reared in fly food with $1.5 \%$ (Fig. 2E) or $3 \%$ GCE (Fig. 2E). Compared to GCE, hydroxycitrate removed calcium oxalate kidney stone formation at 
the concentrations of $0.3 \%, 1.5 \%$ with much less efficiency (Fig.2E). The total stone area decreased by about $50 \%$ in renal tubules of flies reared in fly food with $0.3 \% \mathrm{HCA}$ compared to flies reared in $0.1 \% \mathrm{NaOx}$ only. Citric acid could only partially remove calcium oxalate stone in Drosophila renal tubules at a very high concentration of $3 \%$. The total stone area decreased by $20 \%$ compared to the flies in control group (Fig.1E). Our results strongly suggest that Garcinia cambogia extract could be used to efficiently remove calcium oxalate renal stones in calcium oxalate nephrolithiasis patients.

\section{Garcinia cambogia extract plays a similar role in genetic calcium oxalate kidney stone Drosophila model.}

To test whether Garcinia cambogia extract plays a similar role in genetic nephrolithiasis cases, we first developed a genetic calcium oxalate kidney stone Drosophila model based on published protocol (Hirata et al., 2012b). We reasoned that if Malpighian tubule is an ideal genetic model for nephrolithiasis, RNAi knock-down of nephrolithiasisrelated genes in Malpighian tubule should lead to the formation of calcium oxalate stones. Mutations in the vacuolar-type $\mathrm{H}^{+}$-ATPase (v-ATPase) subunit gene ATP6V1B1 and ATP6V0A4 have been identified in recurrent calcium oxalate kidney stone formers with the highest frequency, indicating that v-ATPase is essential to the formation of calcium oxalate kidney stones (Dhayat et al., 2016). The principal cells are the major Malpighian tubular cell type through which cations are transported. Vha55 and Vha1002 are fly homologs of ATP6V1B1 and ATP6V0A4, and highly expressed in Malpighian tubules (FlyAtlas). UAS-RNAi/Gal4 system has been widely used to knock-down gene expression in a cell- specific manner in Drosophila. To specifically silence Vha55 and 
Vha100-2 genes in Malpighian tubule principal cells, we used the Uro-GAL4 that drives gene expression specifically in the tubular principal cells (Dow and Romero, 2010, Hirata et al., 2012b), and crossed it to the UAS-RNAi lines containing a dsRNA hairpin directed against Vha55 and Vha100-2. We found that RNAi knock-down of vha55 and vha100-2 significantly led to increased formation of calcium oxalate stone in Malpighian tubules compared to control (Uro-Gal4/+)(Figure 3A,D,G). RNAi knockdown of other vATPase subunits also resulted in increased calcium oxalate stone formation (Fig.S1). Control or vha100-2/vha55 RNAi knockdown flies were reared in fly food containing $0.3 \% \mathrm{NaOx}$ for one week. Flies were then transferred to fly food containing $0.1 \% \mathrm{NaOx}$ and different concentration of GCE $(0 \%, 0.5 \%$ and $1 \%)$ for another week, and calcium oxalate stone formation was examined in adult Malpighian tubules. As shown in Figure 3, Garcinia cambogia extract (GCE) removed calcium oxalate stones preformed in renal tubules of vha55/vha100-2 RNAi knockdown flies in a concentration-dependent manner (Fig. 3A-J). The total stone area in renal tubules was significantly decreased to $12.68 \%$ $(0.1 \% \mathrm{NaOx}+0.5 \% \mathrm{GCE})$ and $0 \%(0.1 \% \mathrm{NaOx}+1 \% \mathrm{GCE})$ compared to flies reared in $0.1 \% \mathrm{NaOx}$ only. These results showed that Garcinia cambogia extract efficiently removed calcium oxalate stones in genetic calcium oxalate kidney stone Drosophila model. Altogether, our results strongly suggest that Garcinia cambogia extract could be used to remove calcium oxalate stones in both genetic and non-genetic kidney stone disease models.

Hydroxycitrate efficiently dissolves calcium oxalate stones in Drosophila renal tubules ex vivo. 
We have showed earlier that GCE can remove calcium oxalate stones from renal tubules in vivo. There are two possible pathways for this process: GCE can either facilitate the excretion of stones out of the Malpighian tubule or directly dissolve calcium oxalate stones in situ. To further elucidate the molecular mechanism through which GCE removes calcium oxalate stones from renal tubules, we monitored the dissolution of calcium oxalate stones pre-formed in the Malpighian tubule using live-imaging technique. Renal stone dissolution rate was calculated by dividing the remaining stone area by the total stone area before treatment. As shown in Figure 4, HCA directly dissolved calcium oxalate stones in renal tubules ex vivo in a concentration- and timedependent manner. No stones were excreted from the Malpighian tubule during our observation. Fifty percent of $\mathrm{CaOx}$ stones were dissolved in $0.5 \% \mathrm{HCA}$ solution in 2 hours, and were completely dissolved in 1\% HCA and 1\% CA within 2 hours depending on the original size of stones. There are no difference between H C A AND CA. At the same time, we also monitored the effect of Garcinia cambogia extract on the dissolution of calcium oxalate stones pre-formed in the Malpighian tubule for 3 hours. We did not observe any impact of GCE on the removal of calcium oxalate stones ex vivo, suggesting that the release of free HCA from GCE is essential for its function to dissolve the calcium oxalate stones in vivo. GCE added in fly food could be absorbed in midgut and released into the hemolymph as free HCA form, and then secreted into Malpighian tubule lumen where HCA could dissolve calcium oxalate renal stones. Our results strongly support the idea that Garcinia cambogia extract removes calcium oxalate renal stones via the direct dissolution of stones by HCA in the renal tubules. 


\section{DISCUSSION}

Over the past 30 years, no major progress has been made in novel drug discovery for kidney stone treatment because of the lack of ideal animal models feasible for high-throughput drug screening. In this study, we established a genetic nephrolithiasis Drosophila model which can be used to screen novel drugs for calcium oxalate renal stones. RNAi knock-down of 5 subunits of v-ATPase using UASRNAi/Uro-Gal4 system dramatically enhanced calcium oxalate stone formation in Drosophila Malpighian tubules, suggesting that v-ATPase complex in principal cells is essential for calcium oxalate stone formation and Drosophila is an ideal model to study the molecular function of genes identified from patients with genetic nephrolithiasis.

Garcinia cambogia is a tropical fruit that grows in Southeast Asia and its rind has been widely used for cooking in India. Garcinia cambogia extract containing $60 \%$ hydroxycitrate is a popular weight-loss supplement sold at most health supplement and drug stores. Chung et al has showed that HCA induces dissolution of the calcium oxalate crystal in vitro(Chung et al., 2016). In this study, our data showed that GCE prevented calcium oxalate kidney stone formation in Drosophila renal tubules. We also showed that treatment with GCE completely removed pre-formed calcium oxalate kidney stones from adult Drosophila renal tubules in a concentration-dependent manner. Surprisingly, HCA added in fly food had no effect on the formation and removal of calcium oxalate renal stones in Drosophila Malpighian tubules even at a very high concentration. We speculated that HCA added in fly food could not be absorbed and transported to Malpighian tubules because of the absence of key components in GCE which were essential for the delivery of HCA to Malpighian tubules. Our study also 
showed that GCE was effective in both genetic and non-genetic Drosophila kidney stone models, suggesting that GCE could be a novel medication for the treatment of nephrolithiasis. Nevertheless, our study showed that the Drosophila Malpighian tubule nephrolithiasis model could be used to efficiently screen thousands of traditional Chinese herbal medicine (TCM) to seek the golden treasure of TCM for nephrolithiasis treatment.

Our study also showed that hydroxycitrate could directly dissolve calcium oxalate stones in renal tubules ex vivo in a concentration-dependent manner. The efficiency of HCA dissolving calcium oxalate stones in renal tubule is very high. Calcium oxalate stones in dissected renal tubules were completely dissolved in $0.5 \%$ and $1 \% \mathrm{HCA}$ within 120 minutes. On the other hand, we thought that the HCA concentration in $2 \%$ GCE would be high enough for the dissolution of calcium oxalate stones because GCE contains $60 \%$ HCA. However, $2 \%$ GCE surprisingly had no effect on the dissolution of calcium oxalate stones in Malpighian tubules ex vivo. We reasoned that this was because $31.3 \%$ of GCE is composed of fiber, which cannot be dissolved in water, and as a result, HCA could not be released from GCE water solution. We also tried GCE solution in ethanol, but it also did not dissolve calcium oxalate stones ex vivo. We did not observe any impact of GCE on the removal of calcium oxalate stones ex vivo, suggesting that the release of HCA from GCE is essential for its function to remove the calcium oxalate stones in vivo. GCE added in fly food could be absorbed in midgut and released into hemolymph as free HCA form, and then secreted into Malpighian tubule lumen where HCA could dissolve calcium oxalate renal stones. Our results strongly support the idea that Garcinia cambogia extract removes calcium oxalate renal stones 
via the direct dissolution of stones by HCA in the renal tubules.

Currently potassium citrate is often prescribed to patients with calcium oxalate kidney stone diseases. However, its clinical impact on the removal of kidney stones is still controversial. In this study, we showed that citric acid efficiently dissolved calcium oxalate stone in dissected renal tubules ex vivo, however, the effect of citric acid on calcium oxalate kidney stone is minimal compared to GCE in vivo. To improve the efficiency of citric acid in clinic, we need to find better ways to deliver potassium citrate to patients.

In summary, this work is the first to clearly demonstrate that clinical-grade Garcinia cambogia extract removes calcium oxalate stones in Drosophila Malpighian tubule in vivo and hydroxycitrate can directly dissolve calcium oxalate stones in renal tubules ex vivo. Our study discovered a novel therapeutic strategy for the clinical treatment of nephrolithiasis, and clinical-grade Garcinia cambogia extract could be used to treat patients with nephrolithiasis in the near future.

CONCLUSIONS: Garcinia cambogia extract removes calcium oxalate stones in Drosophila Malpighian tubule, and hydroxycitrate- a major component of GCE, directly dissolve calcium oxalate stones in renal tubules ex vivo. Garcinia cambogia extract has the potential to be used to treat patients with nephrolithiasis.

\section{METHODS}

Fly Strains. Flies were reared on standard food at $25^{\circ} \mathrm{C}$. All UAS-Gal4 crosses were performed at $25^{\circ} \mathrm{C}$. Uro-Gal4 (BI-44416) and UAS-nGFP (Bl-4775) were obtained from 
the Bloomington Drosophila stock center. UAS-RNAi transgenic fly lines targeting vha100-2 (TH04790.N, BI-64859) and vha55 (THU4117 and v-46554), referred in the main text and figures as vha100-2 IR and vha55 IR, were obtained from the Bloomington Drosophila stock center, Vienna stock center and Tsinghua Fly center. Uro-Gal4 and UAS-nGFP were recombined together to label principal cells at all developmental stages.

Chemicals. Potassium hydroxycitrate tribasic monohydrate (59847) and Potassium citrate tribasic monohydrate (c8385) were purchased from Sigma. Swanson ${ }^{\circledR}$ Super Citrimax Clinical Strength Garcinia Cambogia was purchased online from Amazon. Other chemicals were purchased from Sigma unless otherwise indicated.

RNAi knockdown of nephrolithiasis- related genes in principal cells.

This method was adopted from our recent study described previously(Zhang et al., 2013). Briefly, the UAS/Gal4 system allows for the over-expression or knockdown of "gene-of-interest" in a cell- specific manner in Drosophila. A fly line that possesses a "GAL4-driver" can be crossed to a second transgenic fly line containing a construct of interest gene $X$ placed downstream of a UAS promoter sequence. This allows the downstream transgene to be expressed specifically in these cells where GAL4 is expressed. To specifically knock down nephrolithiasis- related genes in Malpighian tubule principal cells, we used the Uro-GAL4 driver, which drives gene expression specifically in the tubular principal cells, and crossed it to the UAS-RNAi lines containing a dsRNA hairpin directed against gene $X$. 
RNAi -based functional analysis of malpighian tubule genes. 10 virgins of UroGal4/UAS-nGFP flies were crossed with 5 males of UAS-RNAi transgenic line in vials at $25^{\circ} \mathrm{C}$. Freshly hatched flies were transferred to fly food with $0.3 \% \mathrm{NaOx}$ at $25^{\circ} \mathrm{C}$ for 1 week. Malpighian tubules were dissected in PBS under dissection microscope and then subjected to the examination of renal stone in malpighian tubules under polarized white light with an Olympus BX63 optical microscope. We randomly selected three images for quantification. Renal stone formation was measured in the whole field of view (700umx100um, 20x magnification) using cellSens software. The results were expressed as mean \pm SD $(n=10)$. All statistical analyses were performed using GraphPad Prism5 software. Statistical significance was defined as $\mathrm{P}<0.05$.

Calcium oxalate stone prevention analysis in vivo. Briefly, wild type or mutant flies were reared on regular fly food containing $0.1 \% \mathrm{NaOx}$ and different concentration of $\mathrm{HCA}$ or GCE at $25^{\circ} \mathrm{C}$ for one week. Malpighian tubules of adult female flies were dissected in PBS under dissection microscope and then subjected to the examination of renal stone in malpighian tubules under polarized white light with an Olympus BX63 optical microscope. We randomly selected three images for quantification. Renal stone formation was measured in the whole field of view (700umx100um, 20x magnification) using cellSens software. The results were expressed as mean \pm SD $(n=10)$. All statistical analyses were performed using GraphPad Prism5 software. Statistical significance was defined as $P<0.05$.

Calcium oxalate stone removal assay in vivo. First, wild type or mutant flies were 
reared on regular fly food containing $0.3 \% \mathrm{NaOx}$ at $25^{\circ} \mathrm{C}$ for one week and renal tubule stone formation was evaluated briefly. Then, flies were transferred to fly food containing $0.1 \% \mathrm{NaOx}$ and different concentration of $\mathrm{HCA}$ or GCE at $25^{\circ} \mathrm{C}$ for 1 week. Malpighian tubules of adult female flies were dissected in PBS under dissection microscope and then subjected to the examination of renal stone in malpighian tubules under polarized white light with an Olympus BX63 optical microscope. We randomly selected three images for quantification. Renal stone formation was measured in the whole field of view (700umx100um, 20x magnification) using cellSens software. The results were expressed as mean \pm SD $(n=10)$. All statistical analyses were performed using GraphPad Prism5 software. Statistical significance was defined as $\mathrm{P}<0.05$.

Ex vivo Calcium oxalate stone dissolution analysis. Briefly, wild type $\mathrm{w}^{1118}$ flies were reared on regular fly food containing $0.3 \% \mathrm{NaOx}$ for one week. Intact Malpighian tubules were dissected in PBS under dissection microscope and transferred onto a slide. 100ul CA, HCA or GCE solution was added to completely cover the MT tissue, and then the Malpighian tubules were subjected to live-imaging under polarized white light with an Olympus BX63 optical microscope without a coverslip. Images were taken every $20 \mathrm{~min}$ and the total area of renal stones was measured using cellSens software. Renal stone dissolution rate was calculated by dividing the remaining stone area by the total stone area at the beginning. The results were expressed as mean $\pm S D(n=5)$. All statistical analyses were performed using GraphPad Prism5 software.

LIST OF ABBREVIATIONS: GCE: Garcinia cambogia extract, HCA: hydroxycitric acid, 
CaOx: calcium oxalate, NaOx: Sodium oxalate, MT: Malpighian tubule

Competing interests: The authors declare that they have no competing interests

\section{Authors' contributions:}

F.J.Z. and F.F.H. designed the study; Q.X.F., X.M.F., X.Z.H, S.Q.G and J.W.T carried out the experiments; Q.X.F., F.F.H and F.J.Z. analyzed the data; Q.X.F. and F.J.Z. made the figures; F.F.H and F.J.Z. drafted and revised the paper; all authors approved the final version of the manuscript.

Acknowledgements: We thank the Bloomington fly stock center and the Tsinghua fly stock center for Drosophila stocks. We want to thank Dr. Haiyan Fu for buying and bringing back GCE reagents from US.

Funding: F.J.Z. was supported by grants from National Key Research Plan (2017YFA0104602) and F.F.H was supported by the National Key Technology Support Program of China (2013BAI09B06 and 2015BAI2B07), the State Key Program of National Natural Science Foundation of China (81430016), the Major International (Regional) Joint Research Project of National Natural Science Foundation of China (81620108003), the Major Scientific and Technological Planning Project of Guangzhou (15020010), and the Guangzhou Clinical Research Center for Chronic Kidney Disease Program (7415695988305). 


\section{References:}

BRAUN, D. A., LAWSON, J. A., GEE, H. Y., HALBRITTER, J., SHRIL, S., TAN, W., STEIN, D., WASSNER, A. J., FERGUSON, M. A., GUCEV, Z., FISHER, B., SPANEAS, L., VARNER, J., SAYER, J. A., MILOSEVIC, D., BAUM, M., TASIC, V. \& HILDEBRANDT, F. (2016), "Prevalence of Monogenic Causes in Pediatric Patients with Nephrolithiasis or Nephrocalcinosis", Clin J Am Soc Nephrol, Vol. 11 No. 4, pp. 664-72.

CHEN, W. C., CHEN, H. Y., LIAO, P. C., WANG, S. J., TSAI, M. Y., CHEN, Y. H. \& LIN, W. Y. (2017), "Toward a new insight of calcium oxalate stones in Drosophila by micro-computerized tomography", Urolithiasis, . CHEN, Y. H., LIU, H. P., CHEN, H. Y., TSAI, F. J., CHANG, C. H., LEE, Y. J., LIN, W. Y. \& CHEN, W. C. (2011), "Ethylene glycol induces calcium oxalate crystal deposition in Malpighian tubules: a Drosophila model for nephrolithiasis/urolithiasis", Kidney Int, Vol. 80 No. 4, pp. 369-77.

CHI, T. \& STOLLER, M. L. (2011), "Stones: Passing a stone in your sleep might be easier than you think", Nat Rev Urol, Vol. 8 No. 10, pp. 533-4.

CHI, T., KIM, M. S., LANG, S., BOSE, N., KAHN, A., FLECHNER, L., BLASCHKO, S. D., ZEE, T., MUTELIEFU, G., BOND, N., KOLIPINSKI, M., FAKRA, S. C., MANDEL, N., MILLER, J., RAMANATHAN, A., KILLILEA, D. W., BRUCKNER, K., KAPAHI, P. \& STOLLER, M. L. (2015), "A Drosophila model identifies a critical role for zinc in mineralization for kidney stone disease", PLoS One, Vol. 10 No. 5, pp. e0124150.

CHUNG, J., GRANJA, I., TAYLOR, M. G., MPOURMPAKIS, G., ASPLIN, J. R. \& RIMER, J. D. (2016), "Molecular modifiers reveal a mechanism of pathological crystal growth inhibition", Nature, Vol. 536 No. 7617, pp. 446-50.

DAGA, A., MAJMUNDAR, A. J., BRAUN, D. A., GEE, H. Y., LAWSON, J. A., SHRIL, S., JOBST-SCHWAN, T., VIVANTE, A., SCHAPIRO, D., TAN, W., WAREJKO, J. K., WIDMEIER, E., NELSON, C. P., FATHY, H. M., GUCEV, Z., SOlimAN, N. A., HASHMI, S., HALBRITTER, J., HALTY, M., KARI, J. A., EL-DESOKY, S., FERGUSON, M. A., SOMERS, M., TRAUM, A. Z., STEIN, D. R., DAOUK, G. H., RODIG, N. M., KATZ, A., HANNA, C., SCHWADERER, A. L., SAYER, J. A., WASSNER, A. J., MANE, S., LIFTON, R. P., MILOSEVIC, D., TASIC, V., BAUM, M. A. \& HILDEBRANDT, F. (2018), "Whole exome sequencing frequently detects a monogenic cause in early onset nephrolithiasis and nephrocalcinosis", Kidney Int, Vol. 93 No. 1, pp. $204-213$.

DHAYAT, N. A., SCHAller, A., AlBANO, G., POINDEXTER, J., GRIFFITH, C., PASCH, A., GALLATI, S., VOGT, B., MOE, O. W. \& FUSTER, D. G. (2016), "The Vacuolar H+-ATPase B1 Subunit Polymorphism p.E161K Associates with Impaired Urinary Acidification in Recurrent Stone Formers", J Am Soc Nephrol, Vol. 27 No. 5, pp. 1544-54.

DOW, J. A. \& ROMERO, M. F. (2010), "Drosophila provides rapid modeling of renal development, function, and disease", Am J Physiol Renal Physiol, Vol. 299 No. 6, pp. F1237-44.

DOWEN, F. E., SAYERS, J. A., HYNES, A. M. \& SAYER, J. A. (2014), "CYP24A1 mutation leading to nephrocalcinosis", Kidney Int, Vol. 85 No. 6, pp. 1475.

GEE, H. Y., JUN, I., BRAUN, D. A., LAWSON, J. A., HALBRITTER, J., SHRIL, S., NELSON, C. P., TAN, W., STEIN, D., WASSNER, A. J., FERGUSON, M. A., GUCEV, Z., SAYER, J. A., MILOSEVIC, D., BAUM, M., TASIC, V., LEE, M. G. \& HILDEBRANDT, F. (2016), "Mutations in SLC26A1 Cause Nephrolithiasis", Am J Hum Genet, Vol. 98 No. 6, pp. 1228-1234.

GEE, H. Y., ZHANG, F., ASHRAF, S., KOHL, S., SADOWSKI, C. E., VEGA-WARNER, V., ZHOU, W., LOVRIC, S., FANG, H., NETTLETON, M., ZHU, J. Y., HOEFELE, J., WEBER, L. T., PODRACKA, L., BOOR, A., FEHRENBACH, H., INNIS, J. W., WASHBURN, J., LEVY, S., LIFTON, R. P., OTTO, E. A., HAN, Z. \& HILDEBRANDT, F. (2015), "KANK deficiency leads to podocyte dysfunction and nephrotic syndrome", $J$ Clin Invest, Vol. 125 No. 6, pp. 2375-84.

HALBRITTER, J., BAUM, M., HYNES, A. M., RICE, S. J., THWAITES, D. T., GUCEV, Z. S., FISHER, B., SPANEAS, L., PORATH, J. D., BRAUN, D. A., WASSNER, A. J., NELSON, C. P., TASIC, V., SAYER, J. A. \& HILDEBRANDT, F. (2015), "Fourteen monogenic genes account for $15 \%$ of nephrolithiasis/nephrocalcinosis", $J$ Am Soc Nephrol, Vol. 26 No. 3, pp. 543-51.

HIRATA, T., CABRERO, P., BERKHOLZ, D. S., BONDESON, D. P., RITMAN, E. L., THOMPSON, J. R., DOW, J. A. \& ROMERO, M. F. (2012a), "In vivo Drosophilia genetic model for calcium oxalate nephrolithiasis", $A m J$ Physiol Renal Physiol, Vol. 303 No. 11, pp. F1555-62.

HIRATA, T., CABRERO, P., BERKHOLZ, D. S., BONDESON, D. P., RITMAN, E. L., THOMPSON, J. R., DOW, J. A. \& ROMERO, M. F. (2012b), "In vivo Drosophilia genetic model for calcium oxalate nephrolithiasis", $A m J$ Physiol Renal Physiol, Vol. 303 No. 11, pp. F1555-62.

HO, C. Y., CHEN, Y. H., WU, P. Y., CHANG, C. H., CHEN, H. Y., MAN, K. M., SHEN, J. L., TSAI, F. J., LIN, W. Y., LEE, Y. J. \& CHEN, W. C. (2013), "Effects of commercial citrate-containing juices on urolithiasis in a 
Drosophila model", Kaohsiung J Med Sci, Vol. 29 No. 9, pp. 488-93.

KNAUF, F. \& PREISIG, P. A. (2011), "Drosophila: a fruitful model for calcium oxalate nephrolithiasis?", Kidney Int, Vol. 80 No. 4, pp. 327-9.

LANDRY, G. M., HIRATA, T., ANDERSON, J. B., CABRERO, P., GALLO, C. J., DOW, J. A. \& ROMERO, M. F. (2016), "Sulfate and thiosulfate inhibit oxalate transport via a dPrestin (Slc26a6)-dependent mechanism in an insect model of calcium oxalate nephrolithiasis", Am J Physiol Renal Physiol, Vol. 310 No. 2, pp. F152-9.

MILlER, J., CHI, T., KAPAHI, P., KAHN, A. J., KIM, M. S., HIRATA, T., ROMERO, M. F., DOW, J. A. \& STOLLER, M. L. (2013), "Drosophila melanogaster as an emerging translational model of human nephrolithiasis", $J$ Urol, Vol. 190 No. 5, pp. 1648-56.

RAMASWAMY, K., KILLILEA, D. W., KAPAHI, P., KAHN, A. J., CHI, T. \& STOLLER, M. L. (2015), "The elementome of calcium-based urinary stones and its role in urolithiasis", Nat Rev Urol, Vol. 12 No. 10, pp. 543-57.

SAYER, J. A. (2017), "Progress in Understanding the Genetics of Calcium-Containing Nephrolithiasis", J Am Soc Nephrol, Vol. 28 No. 3, pp. 748-759.

WU, S. Y., SHEN, J. L., MAN, K. M., LEE, Y. J., CHEN, H. Y., CHEN, Y. H., TSAI, K. S., TSAI, F. J., LIN, W. Y. \& CHEN, W. C. (2014), "An emerging translational model to screen potential medicinal plants for nephrolithiasis, an independent risk factor for chronic kidney disease", Evid Based Complement Alternat Med, Vol. 2014972958.

ZHANG, F. \& CHEN, X. (2014), "The Drosophila nephrocyte has a glomerular filtration system", Nat Rev Nephrol, Vol. 10 No. 9, pp. 491.

ZHANG, F., ZHAO, Y. \& HAN, Z. (2013), "An in vivo functional analysis system for renal gene discovery in Drosophila pericardial nephrocytes", J Am Soc Nephrol, Vol. 24 No. 2, pp. 191-7.

ZHANG, F., ZHAO, Y., CHAO, Y., MUIR, K. \& HAN, Z. (2013), "Cubilin and amnionless mediate protein reabsorption in Drosophila nephrocytes", J Am Soc Nephrol, Vol. 24 No. 2, pp. 209-16. 


\section{Figure Legends}

Figure1. Garcinia cambogia extract prevents calcium oxalate kidney stone formation in Drosophila renal tubules. Representative images of the effect of GCE on the formation of $\mathrm{CaOx}$ stone in adult malpighian tubules (A-D). Calcium oxalate kidney stone formation in wild type flies reared in fly food containing $0.1 \% \mathrm{NaOx}(\mathrm{A})$, $0.1 \% \mathrm{NaOx}+0.3 \%$ GCE (B), $0.1 \% \mathrm{NaOx}+1.5 \%$ GCE (C), $0.1 \% \mathrm{NaOx}+3 \%$ GCE (D). 10 pairs of Malpighian tubules from 5 flies per genotype were dissected and analyzed. Total area of $\mathrm{CaOx}$ stones in Malpighian tubule was measured in the whole field of view (700umx100um, $20 \times$ magnification). The results are expressed as mean \pm SD. Oneway ANOVA was performed to analyze the data and Bonferroni's multiple comparison was performed to compare all pairs of columns. Statistical significance was defined as $p<0.05\left({ }^{*} p<0.05,{ }^{* *} p<0.005,{ }^{* * *} p<0.0005\right)$. (E) Comparison of the effect of GCE, HCA and CA on calcium oxalate kidney stone formation. GCE totally prevented calcium oxalate kidney stone formation in adult renal tubules at $0.3 \%, 1.5 \%$ and $3 \%$ compared to control $\left({ }^{* * *} p<0.0001\right)$; whereas, $3 \%$ of HCA or CA only partially prevented calcium oxalate kidney stone formation compared to control $\left({ }^{* *} p<0.0001\right) .0 .3 \%$ of GCE is more efficient to prevent calcium oxalate kidney stone formation compared to $3 \%$ of $\mathrm{HCA}$ or $\mathrm{CA}\left({ }^{* * *} \mathrm{p}<0.0001\right)$.

Figure2. Garcinia cambogia extract completely removes calcium oxalate kidney stones from Drosophila renal tubules in vivo. Representative images of the effect of GCE on the removal of $\mathrm{CaOx}$ stone in adult Malpighian tubules (A-D). Wild type flies 
were reared in fly food containing $0.3 \% \mathrm{NaOx}$ for one week and then transferred to new fly food containing $0.1 \% \mathrm{NaOx}(\mathrm{A}), \quad 0.1 \% \mathrm{NaOx}+0.1 \%$ GCE (B), $0.1 \% \mathrm{NaOx}+0.5 \%$ GCE (C), $0.1 \% \mathrm{NaOx}+1 \%$ GCE (D) for one week. 10 pairs of Malpighian tubules from 5 flies per genotype were dissected and analyzed. Total area of $\mathrm{CaOx}$ stones in Malpighian tubule was measured in the whole field of view (700umx100um, $20 x$ magnification). The results are expressed as mean \pm SD. One-way ANOVA was performed to analyze the data and Bonferroni's multiple comparison was performed to compare all pairs of columns. Statistical significance was defined as $p<0.05\left({ }^{*} p<0.05\right.$, $\left.{ }^{* *} p<0.005,{ }^{* * *} p<0.0005\right)$. (E) Comparison of the effect of GCE, HCA and CA on calcium oxalate kidney stone removal. Almost all calcium oxalate kidney stones in adult renal tubules were removed in the presence of $1.5 \%$ or $3 \%$ GCE compared to control $\left({ }^{* * *} p<0.0001\right)$, whereas, only $40 \%$ of calcium oxalate kidney stones were removed even in the presence of $3 \% \mathrm{HCA}$ or $\mathrm{CA}\left({ }^{* *} \mathrm{p}<0.005\right.$ or $\left.{ }^{*} \mathrm{p}<0.05\right) .0 .3 \%$ of GCE is more efficient to remove calcium oxalate kidney stone formation compared to $3 \%$ of $\mathrm{HCA}$ or $\mathrm{CA}$ $\left({ }^{* * *} p<0.0001\right)$

Figure3. Garcinia cambogia extract efficiently removes calcium oxalate kidney stones in genetic nephrolithiasis Drosophila model. Representative images of the effect of GCE on the removal of $\mathrm{CaOx}$ stone in adult Malpighian tubules of control UroGal4/+ (A-C), Uro-Gal4/vha55 RNAi knockdown (D-F) and Uro-Gal4/vha100-2 RNAi knockdown flies (G-I). Flies reared in fly food containing $0.3 \% \mathrm{NaOx}$ for one week were transferred to food containing $0.1 \% \mathrm{NaOx}, 0.1 \% \mathrm{NaOx}+0.5 \% \mathrm{GCE}, 0.1 \% \mathrm{NaOx}+1 \%$ GCE for one week. 10 pairs of Malpighian tubules from 5 flies per genotype were 
dissected and analyzed. Total area of $\mathrm{CaOx}$ stones in Malpighian tubule was measured in the whole field of view (700umx100um, $20 \times$ magnification). The results are expressed as mean \pm SD. Two-way ANOVA grouped analyses was performed to analyze the data and Bonferroni's post-test was performed to compare replicate means by row. Statistical significance was defined as $p<0.05 \quad\left({ }^{*} p<0.05,{ }^{* *} p<0.005\right.$, $\left.{ }^{* * *} p<0.0005\right)$. (J). Comparison of the effect of GCE on the removal of calcium oxalate kidney stones in adult Malpighian tubules of control, vha55 RNAi knockdown and vha100-2 RNAi knockdown flies. RNAi knock-down of vha55 and vha100-2 led to increased formation of calcium oxalate stone in Malpighian tubules compared to control (Uro-Gal4/+)( $\left.{ }^{* * *} \mathrm{p}<0.0001\right)$. All calcium oxalate kidney stones in adult renal tubules were removed in the presence of $1 \%$ GCE in all three groups.

Figure4. Hydroxycitrate efficiently dissolves calcium oxalate stones in Drosophila renal tubules ex vivo. Live images of renal stone dissolution in intact Malpighian tubules treated with HCA solutions ex vivo at different time points (A-F). Wild type flies were reared in fly food containing $0.3 \% \mathrm{NaOx}$ for one week. Intact Malpighian tubules were dissected from wild type flies reared in fly food containing $0.3 \% \mathrm{NaOx}$ and treated with HCA, CA or GCE solutions, and renal stone dissolution was monitored using liveimaging. Images were taken at $0 \mathrm{~min}(A), 20 \mathrm{~min}(B), 40 \mathrm{~min}(C), 60 \mathrm{~min}(\mathrm{D}), 100 \mathrm{~min}(\mathrm{E})$, 120 min $(F)$. The total area of renal stones was measured using cellSens software. Renal stone dissolution rate was calculated by dividing the remaining stone area by the total stone area at the beginning. The results are expressed as mean $\pm S D(n=3)$. (G). HCA and CA efficiently dissolved calcium oxalate stones within 120 minutes. Similar to 
control group (H2O), GCE had no effect on dissolving calcium oxalate stones. Same scale bars were used in A-F (100um).

FigureS1. The effects of fly orthologs of 6 mammalian v-ATPase genes on CaOx stone formation in Malpighian tubules. RNAi Knock-down of each gene of v-ATPase complex led to increased formation of calcium oxalate stone in Drosophila Malpighian tubule. The results are expressed as mean \pm SD. The unpaired T-test was performed with two-tailed p-values and $95 \%$ confidence intervals. Statistical significance was defined as $P<0.05 .\left({ }^{*} p<0.05,{ }^{* *} p<0.005,{ }^{* * *} p<0.0005\right)$. 


\section{Figure1}
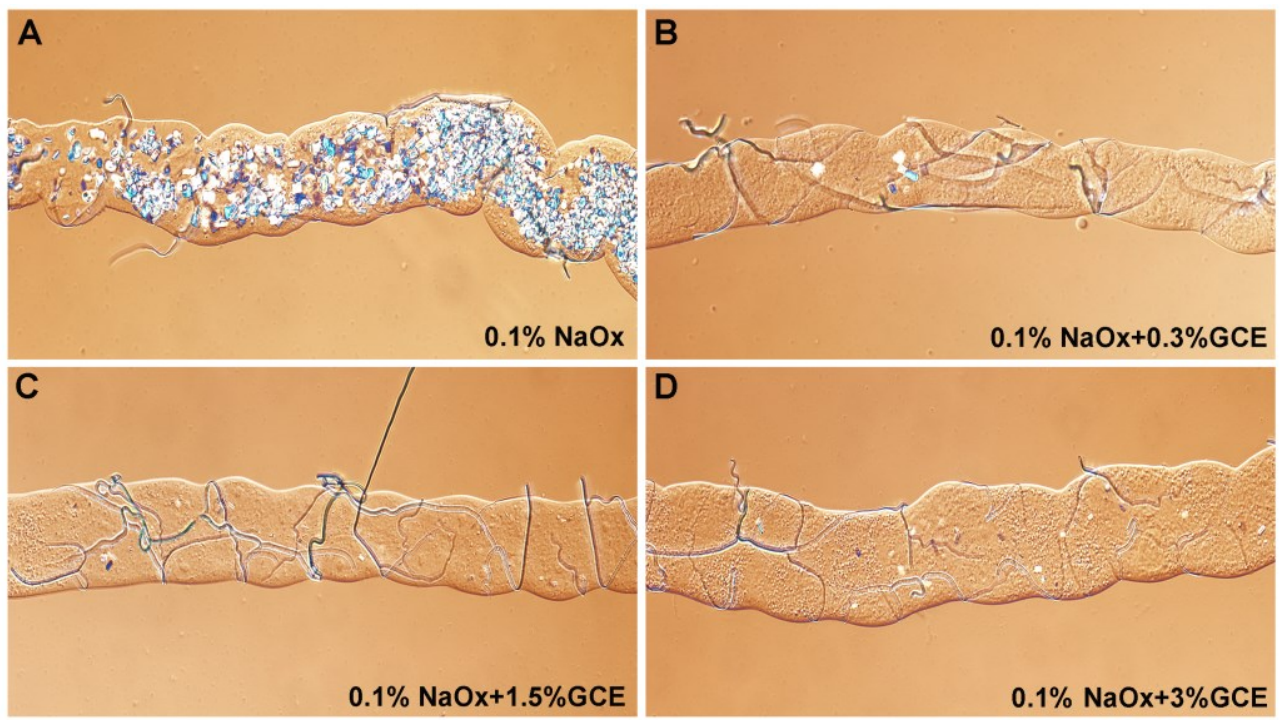

E

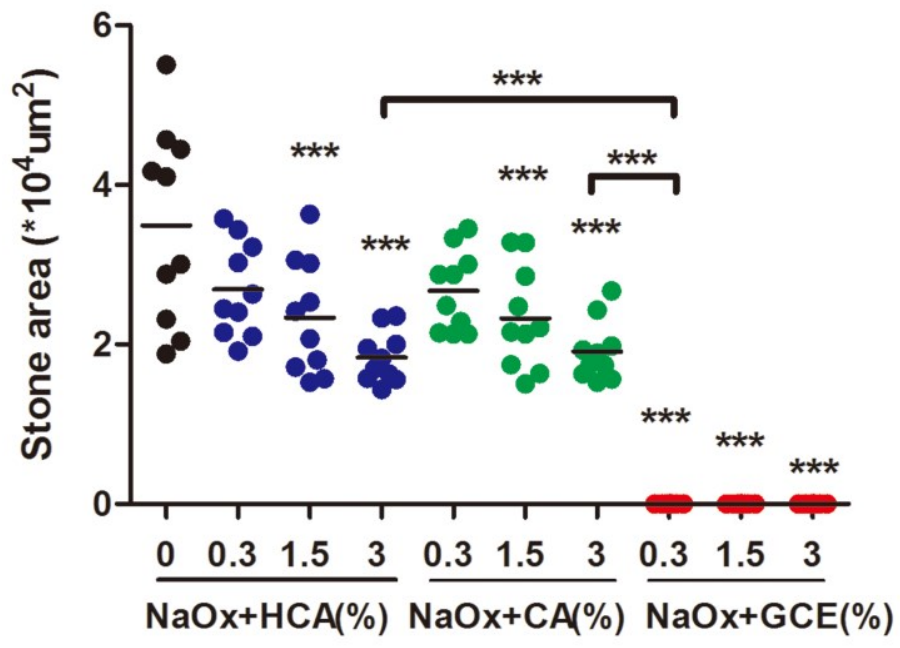




\section{Figure2}
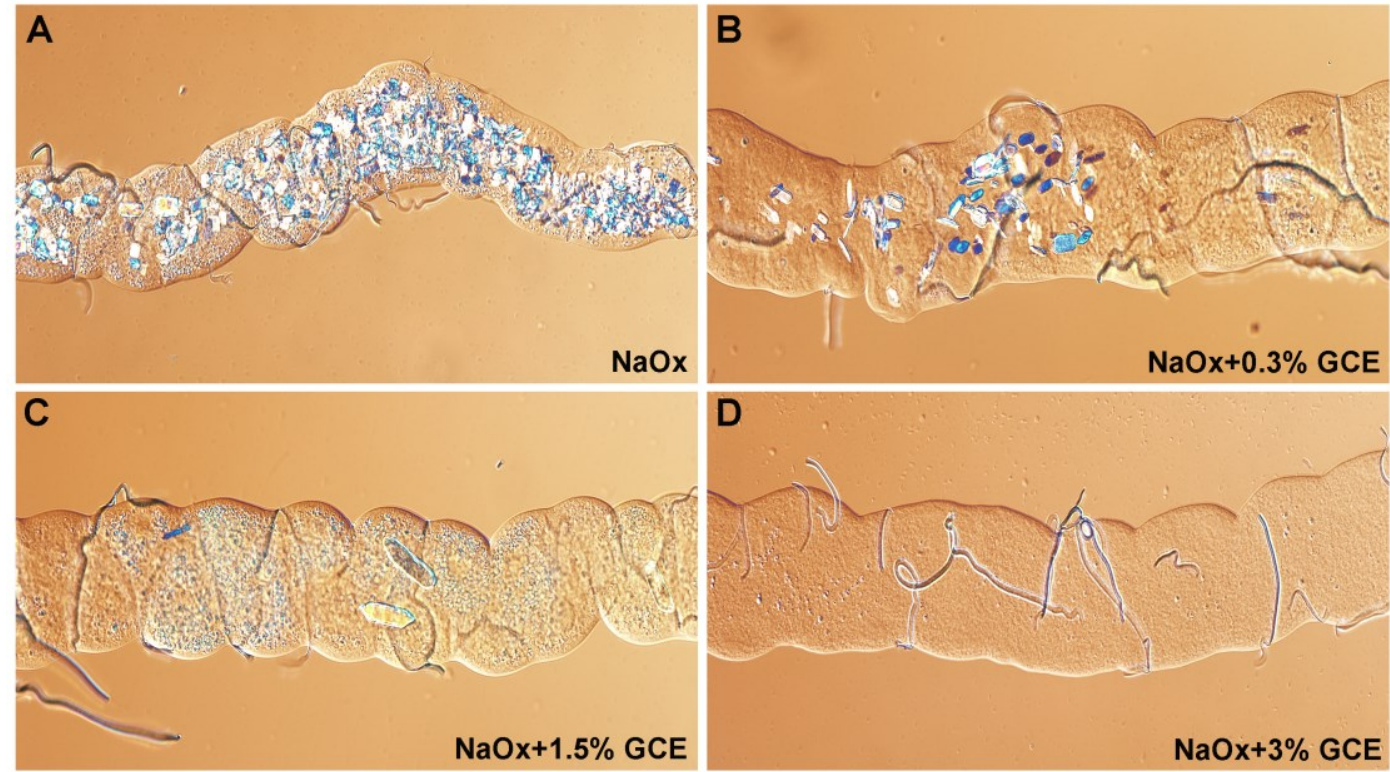

E

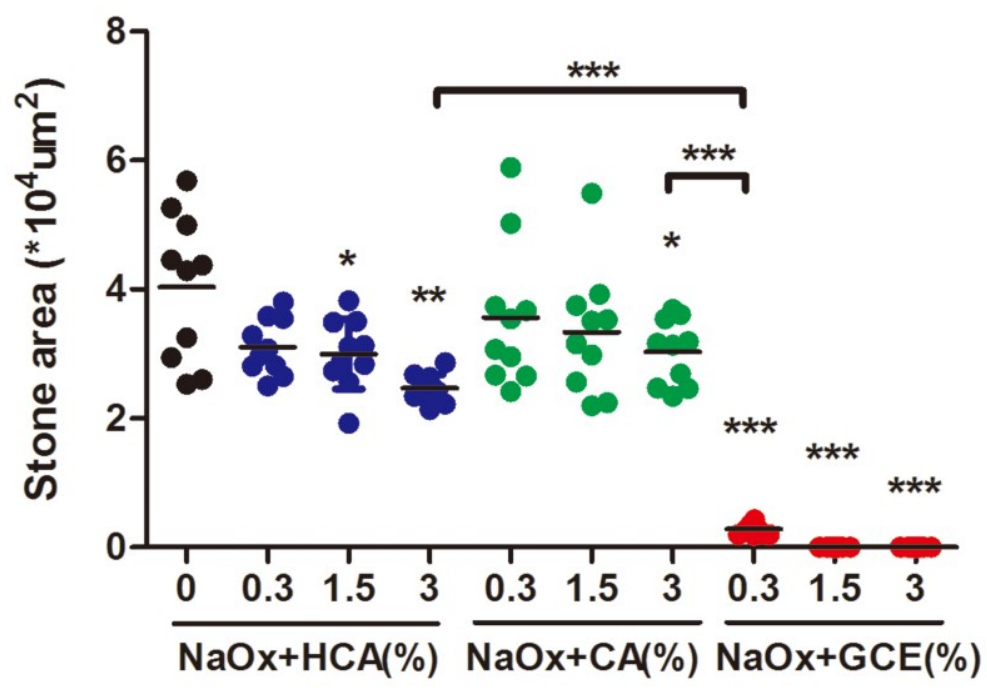




\section{Figure3}

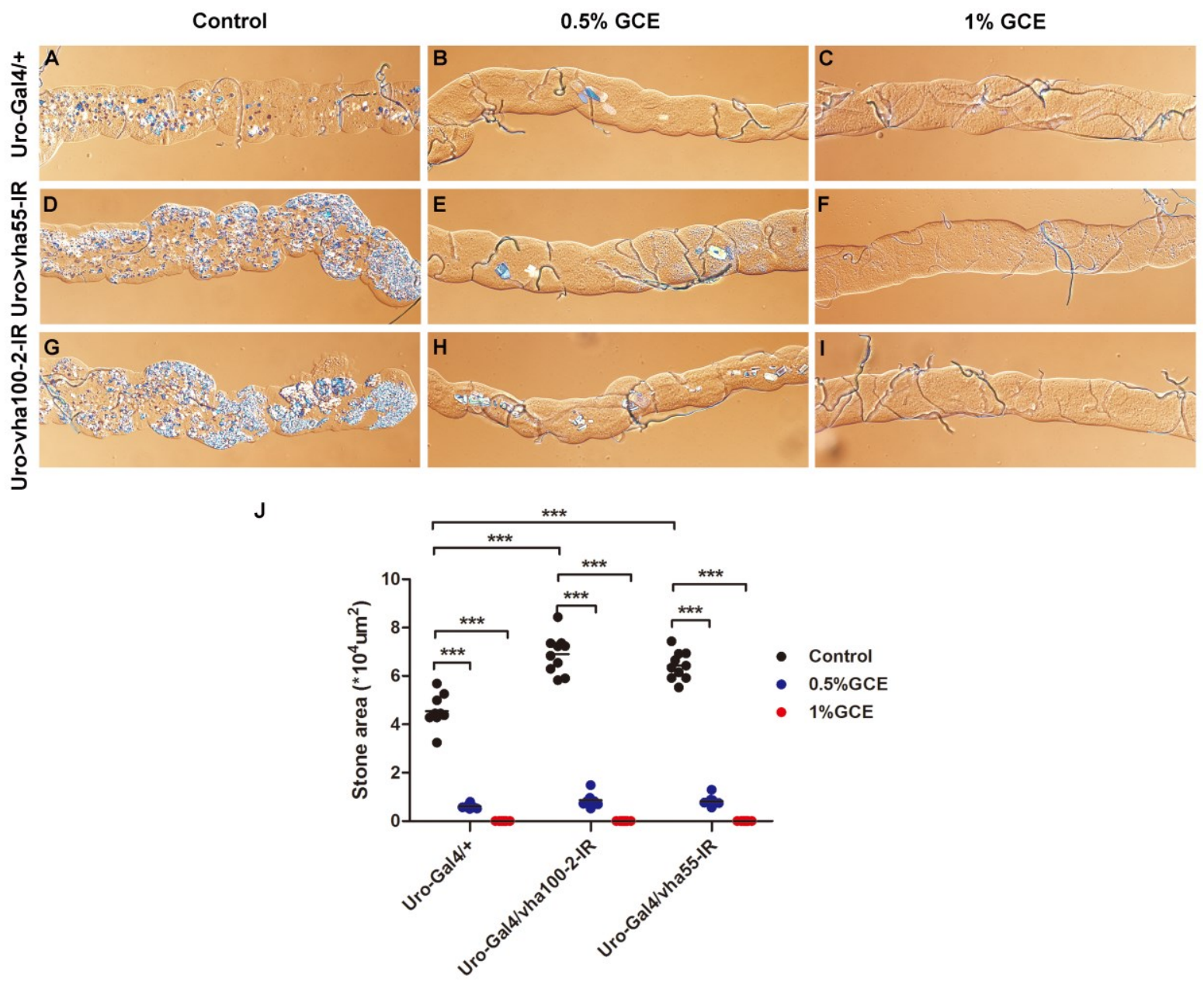




\section{Figure4}
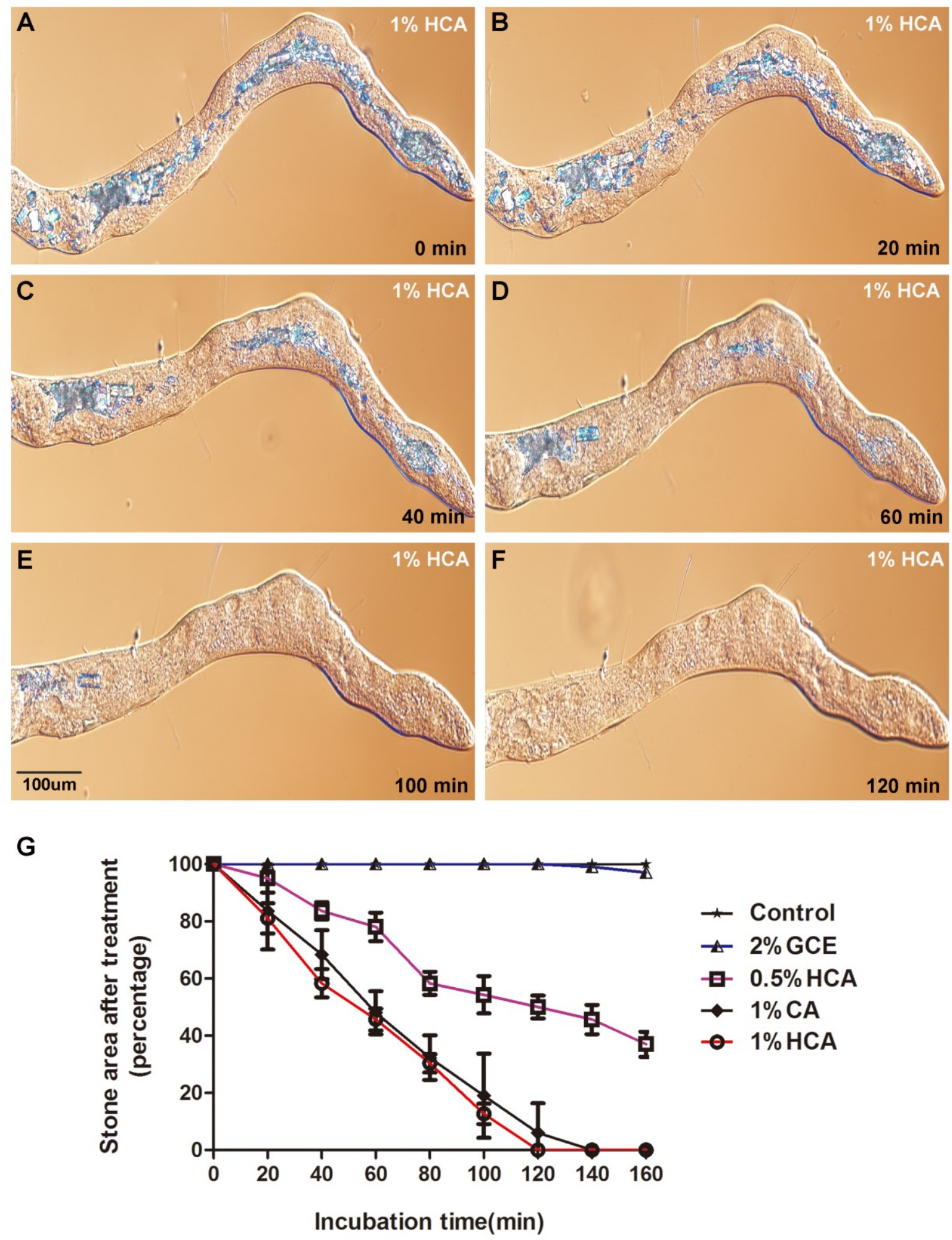
FigureS1

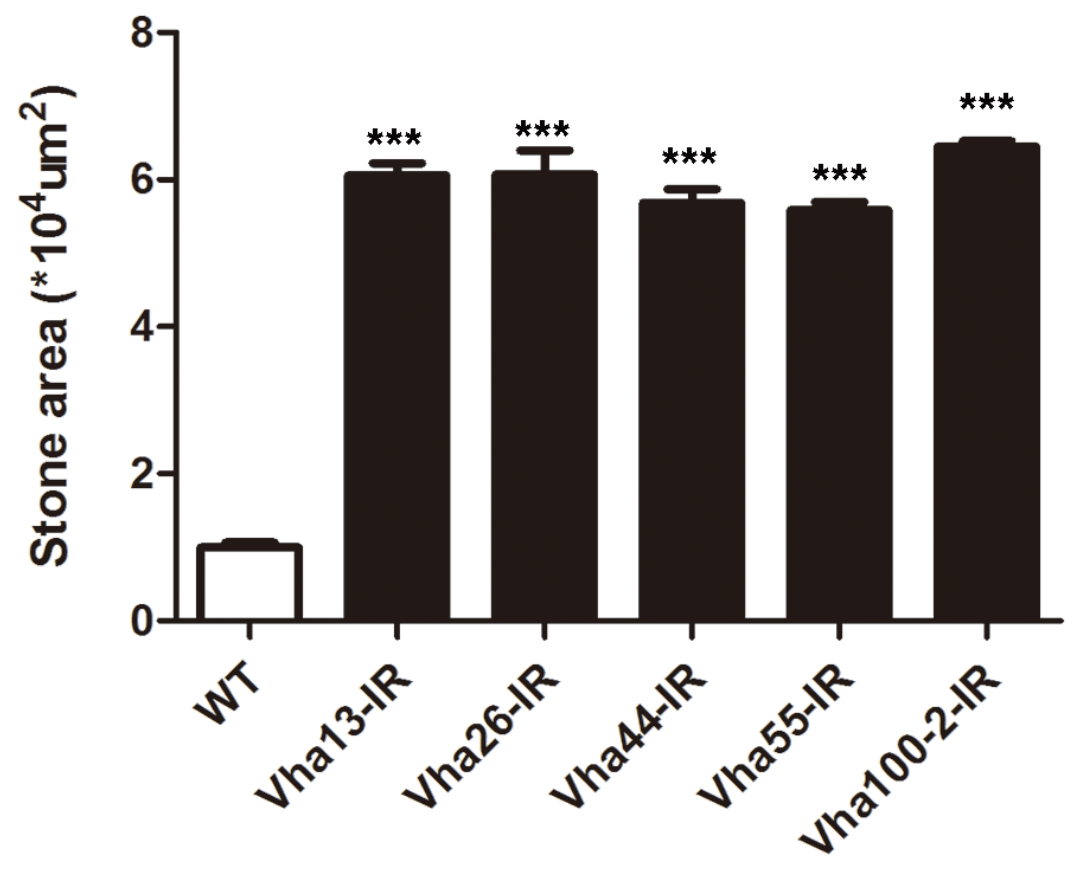

